\title{
Transposition under various combinations of discriminanda'
}

\section{Abstraet}

To test Spence's analysis of transposition, six groups of rats were trained in a two choice discrimination box under various combinations of discriminanda and tested under 21 new relationships. The data, in general, were compatible with the predictions made from the theory.

\section{Problem}

Recently Meyer (1964) reported that the ordinal relationship of the rates of learning under various combinations of discriminanda was compatible with the summation gradient hypothesis. The hypothesis under study is the testing of Spence's analysis of transposition $(1936,1937,1942,1952)$ under these various combinations of discriminanda.

\section{Subjeets}

Sixty male Long-Evans rats, approximately 90 days old at the beginning of the experiment were used as Ss.

\section{Apparatus}

The Ss were trained in a simple flat black, two choice Grice discrimination box. A 4 inch wide starting alley opened into a V-shaped choice chamber that was 12 inches wide. A sliding door separated the alley and choice chamber, and sliding doors could also be lowered in front of the stimuli.

The stimuli, made from Plexiglas, were seven white circles of equal ratio approximately 1.50:1.00 of area size on black background. The diameters of the stimulus circles were 1.32 inches (1), 1.62 inches (2), 1.99 inches (3), 2.44 inches (4), 2.99 inches (5), 3.66 inches (6), and 4.48 inches (7). In the center of each stimulus was a circular door, 1.25 inches in diameter, hinged from the top so that it could be swung open or locked. Behind the stimulus door was a food cup. The stimuli could be shifted with ease from one side of the box to the other. When closed the door and the circle presented a flat surface.

\section{Procedure}

Prediscrimination training. Ss were placed on a daily 23-hour food deprivation schedule for 1 week. On the last 3 days of this period each $\mathrm{S}$ was handled for 10 minutes before feeding. On the following day, the Ss were given 10 minutes of free exploration of the apparatus, followed by 1 hour of eating. During the next session Ss were trained to push open the stimulus doors to get a food reward. During this training the stimuli ( +) later to be used in the discrimination trials were used. On the first trial the stimulus door was held open and on successive trials, the door was closed a little more so that by the eighth trial Ss had learned to push open the door. The side of the $(+)$ stimulus varied in the order
LRLRLRLRLR. The other stimulus door was always obscured by the sliding door in front of the stimulus. The reward was a food pellet of lab chow weighing approximately .55 grams. If the $\mathrm{S}$ did not learn to open the door by the end of the 10 trials it was dropped from the group. One $\mathrm{S}$ was dropped and not replaced from Group I, II, III, and V.

Discrimination training. On the following days, Ss received 10 trials a day until the $\mathrm{S}$ had mastered the problem. The criterion used was 9 or more correct choices from the 10 trials for 3 consecutive days. A noncorrective procedure was used throughout the experiment.

Experimental groups. The 60 Ss were randomly divided into 6 experimental groups. Group I was assigned $\mathrm{a}+$ - discrimination problem where a response to stimulus $2(+)$ was rewarded and a response to stimulus 4 (-) was not. The stimuli for all experimental groups were randomly assigned for each trial to a right or left position. Group II, - + was a replication of Group I with the exception that responses to stimulus $4(+)$ were rewarded and to stimulus $2(-)$ were not. Group III, - + - , where responses made to stimuli 2 and $6(-)$ were not rewarded, whereas those to $4(+)$ were. For Group IV, +-- , responses to $2(+)$ were rewarded and to 4 and 6 (-) were not; in Group $\mathrm{V},+-+$, responses to stimuli 2 and $6(+)$ were associated with rewards and $4(-)$ was not; in Group VI, + + -, responses to 2 and $4(+)$ were rewarded and responses to $6(-)$ were not. For Groups III and IV, the rewarded stimulus (+) was randomly paired with nonrewarded stimuli (-); for Groups V and VI the negative was randomly paired with the rewarded stimuli. No pair of stimuli was presented more than twice in succession.

Transposition trials. After mastering the assigned discrimination problem, the Ss were tested on sets of 21 new relationships as seen from Table I. Three test trials were given per day and were randomly presented among seven discrimination trials. The three test pair of stimuli per day were randomly selected from the 21 relationships as was the order of the pair. No test stimulus pair was presented more than once per $\mathrm{S}$. The response to all the test stimuli were rewarded.

\section{Results and Diseussion}

Transposition as measured by the frequency of response choice to the test stimuli is shown in Table I. Inspection of this table shows that the data are for the most part compatible with Spence's analysis in that for the entire set of test stimuli for the six experimental groups, $81 \%$ of the Ss' choices were predicted from the 


\begin{tabular}{|c|c|c|c|c|c|c|c|c|c|c|c|c|}
\hline $\begin{array}{l}\text { Test stimuli } \\
\text { pair for } \\
\text { transposition }\end{array}$ & $\begin{array}{l}\text { Grou } \\
+2\end{array}$ & ${ }_{4} I$ & $\begin{array}{l}\text { Gro } \\
-2\end{array}$ & $\frac{p}{4}$ II & $\begin{array}{l}\text { Group } \\
-2+4\end{array}$ & $\frac{\text { III }}{6}$ & $\begin{array}{l}\text { Grour } \\
+2\end{array}$ & ${ }_{-6}^{I V}$ & Group +2 & $\begin{array}{l}V \\
+6\end{array}$ & $\begin{array}{l}\text { Grour } \\
+2+4\end{array}$ & $\begin{array}{l}\mathrm{p} \\
4-6\end{array}$ \\
\hline & 9 & p & 9 & p & f & $\bar{p}$ & 1 & p & I & $\mathbf{p}$ & 1 & p \\
\hline $1 / 2$ & 4 & 1 & 3 & 2 & 5 & 2 & 6 & 1 & 3 & 1 & 6 & 2 \\
\hline $1 / 3$ & 4 & 1 & 7 & 3 & 9 & 3 & 4 & 1 & 0 & 1 & 4 & 3 \\
\hline $1 / 4$ & 1 & 1 & 9 & 4 & 9 & 4 & 0 & 1 & 0 & 1 & 4 & 4 \\
\hline $1 / 5$ & 2 & 1 & 9 & 5 & 8 & 5 & 1 & 1 & 3 & 1 & 2 & 1 \\
\hline $1 / 6$ & 3 & 1 & 9 & 6 & 5 & 6 & 0 & 1 & 4 & 1 & 0 & 1 \\
\hline $1 / 7$ & 4 & 1 & 9 & 7 & 4 & $=$ & 0 & 1 & 9 & - & 0 & 1 \\
\hline $2 / 3$ & 1 & 2 & 9 & 3 & 8 & 3 & 2 & 2 & 2 & 2 & 5 & 3 \\
\hline $2 / 4$ & 0 & 2 & 9 & 4 & 9 & 4 & 0 & 2 & 0 & 2 & 4 & 2 \\
\hline $2 / 5$ & 1 & 2 & 7 & 5 & 8 & 5 & 2 & 2 & 1 & 2 & 0 & 2 \\
\hline $2 / 6$ & 0 & 2 & 9 & 6 & 5 & $=$ & 0 & 2 & 6 & $=$ & 0 & 2 \\
\hline $2 / 7$ & 3 & 2 & 9 & 7 & 4 & 2 & 1 & 2 & 7 & 7 & 0 & 2 \\
\hline $3 / 4$ & 2 & 3 & 6 & 4 & 4 & 4 & 0 & 3 & 2 & 3 & 5 & 3 \\
\hline $3 / 5$ & 5 & 3 & 8 & 5 & 4 & $=$ & 0 & 3 & 6 & $=$ & 0 & 2 \\
\hline $3 / 6$ & 4 & 3 & 6 & 6 & 2 & 3 & 0 & 3 & 9 & 6 & 1 & 3 \\
\hline $3 / 7$ & 9 & 3 & 7 & 7 & 1 & 3 & 2 & 3 & 8 & 7 & 0 & 3 \\
\hline $4 / 5$ & 3 & 4 & 7 & 5 & 1 & 4 & 4 & 4 & 6 & 5 & 3 & 4 \\
\hline $4 / 6$ & 6 & 4 & 8 & 6 & 0 & 4 & 2 & 4 & 9 & 6 & 0 & 4 \\
\hline $4 / 7$ & 6 & 4 & 5 & 7 & 1 & 4 & 3 & 4 & 9 & 7 & 1 & 4 \\
\hline $5 / 6$ & 5 & 5 & 7 & $\cong$ & 2 & 5 & 4 & $\cong$ & 5 & 6 & 0 & 5 \\
\hline $5 / 7$ & 7 & 5 & 5 & $\cong$ & 2 & 5 & 6 & $\cong$ & 9 & 7 & 2 & 5 \\
\hline \multirow[t]{2}{*}{$6 / 7$} & 6 & $\cong$ & 4 & $\cong$ & 5 & 6 & 8 & $\cong$ & 8 & 7 & 7 & $\cong$ \\
\hline & \multicolumn{2}{|c|}{$\mathrm{N}=9$} & \multicolumn{2}{|c|}{$\mathrm{N}=9$} & \multicolumn{2}{|c|}{$\mathrm{N}=9$} & \multicolumn{2}{|c|}{$N=10$} & \multicolumn{2}{|c|}{$\mathrm{N}=9$} & \multicolumn{2}{|c|}{$\mathrm{N}=10$} \\
\hline Total & \multicolumn{2}{|c|}{$\%=68$} & \multicolumn{2}{|c|}{$\not x_{0} 88$} & \multicolumn{2}{|c|}{$x=80$} & \multicolumn{2}{|c|}{$\not 6-85$} & \multicolumn{2}{|c|}{$\not b=84$} & \multicolumn{2}{|c|}{$x=81$} \\
\hline
\end{tabular}

theory. As no form of the generalization gradients can be specified, it was not possible to make exact predictions of behavior in the transposition tests. However, general approximations were made using and modifying Spence's (1942) hypothetical generalization curves.

Sixty eight percent of the responses to the test stimuli may be predicted by the theory for Group I, +-, and $88 \%$ for Group II, - +. Combinations of stimuli where transposition broke down may be observed from the data for Groups I and II as transposition pairs became markedly different from the discrimination training stimuli. Disruption of the predictions took place.

Similarly, the experimental results for the four discriminanda groups are supportive of the absolute stimulus analysis. Group III, - + -, was essentially a replication of Spence's research on the intermediate size problem. The data from this group verifies the previous work as $80 \%$ of the responses to the test stimuli fit the theory's predictions. Furthermore, in Group IV, +---, $85 \%$ of the responses are compatible with the analysis; in Group $\mathrm{V},+-+, 84 \%$ of the responses and in Group $\mathrm{VI},++-, 81 \%$. In general, errors were from those pairs of stimuli that were close together. The data, thus, permits the analysis of transposition theorized by Spence to new combinations of discriminanda.

\section{References}

MEYER, M. E. Discrimination learning under various combinations of discriminanda. J. comp. physiol. Psychol., 1964, in press.

SPENCE, K. W. The nature of discrimination learning in animals. Psychol. Rev., 1936, 43, 427-449.

SPENCE, K. W. The differential response in animals to stimuli varying within a single dimension. Psychol. Rev., 1937, 44, 430-444.

SPENCE, K. W. The basis of solution by chimpanzees of the intermediate size problem. J. exp. Psychol., 1942, $31,257-271$.

SPENCE, K. W. The nature of respgnse in discrimination learning. Psychol. Rev., 1952, 59, 89-93.

\section{Note}

1. This research was supported by funds provided by Grant M-6574A from the National Institute of Mental Health, United States Public Health Service. 